\title{
O POSSÍVEL USO DA LATANOPROSTA NA TERAPIA CAPILAR
}

\author{
THE POSSIBLE USE OF LATANOPROST IN HAIR THERAPY
}

\author{
Sarah Ruhama Maciel Freire Santos (SANTOS, S.R.M.F.) \\ Acadêmica do Curso de Biomedicina da FACER - Faculdade Evangélica de Ceres - GO, \\ Brasil.sarahruhama15@hotmail.com
}

\author{
Hueiwida Stefani Barbosa (BARBOSA, H.S.) \\ Acadêmica do Curso de Biomedicina da FACER - Faculdade Evangélica de Ceres - GO, \\ Brasil. hueiwida@hotmail.com
}

\section{Larisse Silva Dalla Libera (LIBERA, L.S.D.)}

Mestre em Ciências da Saúde em ênfase em patologia clínica e doenças humanas pelo programa de Pós-Graduação em Ciências da SaúdeUniversidade Federal de Goiás. Docente da Faculdade Evangélica de Ceres. Goiânia-GO, Brasil. larisse.dalla@gmail.com

\section{Endereço para correspondência:}

Av. Brasil, S/n, Qd. 13, Morada Verde, Ceres-GO, Brasil

CEP:76300-000

Fone/Fax: (62) 3323-1040

Email:sarahruhama15@hotmail.com

\section{RESUMO}

INTRODUÇÃO: A latanoprosta é um análogo à prostaglandinas utilizadas no tratamento de glaucoma, mas que tem sido usado para avaliação da hipertricose. OBJETIVO: Revisar através da literatura o papel da latanoprosta no crescimento de pelos. METODOLOGIA: Trata-se de uma revisão descritiva que avaliou publicações indexadas na base de dados MEDLINE, consultadas por meio do PUBMED; Biblioteca Virtual de Saúde (BVS), Periódicos Capes e Scientific Eletronic Library Online (SCIELO), nos meses de junho e julho de 2018. A partir das análises foram incluídos apenas trabalhos que fizeram uso da latanoprosta em humanos. RESULTADO E DISCUSSÃO: De 111 artigos relacionados com a latanoprosta e o crescimento de pelos, apenas nove foram incluídos. A maioria dos estudos eram do tipo caso-controle com avaliação do uso da latanoprosta principalmente no crescimento de pelos do couro cabeludo e cílios tratados para alopecia areata e androgenética. O número de participantes dos estudos variou de 1 a 54 indivíduos. A latanoprosta foi utilizada principalmente por via tópica na concentração de $0,005 \%$. Infelizmente nem todos os trabalhos incluídos avaliaram a eficácia da latanoprosta ou descreveram adequadamente a maneira como foi investigado o crescimento dos pelos. CONCLUSÃO: Apesar de não ser 
possível afirmar que a latanoprosta é eficaz como terapia capilar, este estudo é promissor e pode apoiar novos trabalhos que investiguem melhor a eficácia e segurança da latanoprosta, bem como a dose ideal e duração do tratamento dela ou de outro candidato análogo das prostaglandinas.

Palavra-chave: Alopecia. Alopecia Androgenética. Prostaglandinas. Latanoprosta

\begin{abstract}
INTRODUCTION: Latanoprost is an analogue of prostaglandins used in treatment of glaucoma, but has been used to evaluation of hypertrichosis. OBJECTIVE: This study through literature the role of latanoprost in the growth of hairs. METHODOLOGY: This is a descriptive review that evaluated publications indexed in the MEDLINE database, consulted through PUBMED; Virtual Health Library (VHL), Periodicals Capes and Scientific Eletronic Library Online (SCIELO) between June and July 2018. All the results were analyzed and included only studies that have used latanoprost in humans. RESULTS AND DISCUSSION: Among the 111 articles related to latanoprost and hair growth, only nine were included. Most of the studies were of the case-control type with evaluation of the use of latanoprost mainly in the growth of scalp hairs and eyelashes treated for alopecia areata and androgenetic. The number of study participants varied from 1- 54 subjects and the medication was mainly used topically at the concentration of $0.005 \%$. Unfortunately, not all the studies included evaluated the efficacy of latanoprost or adequately described the way in which hair growth was evaluated. CONCLUSION: Although not possible to state that latanoprost is effective as a capillary therapy, however this study is promising and may support new studies that had better investigate the efficacy and safety of latanoprost, as well as the optimal dose and duration of treatment another similar candidate in the prostaglandins.
\end{abstract}

Keywords: Alopecia. Alopecia Androgenetic. Prostaglandins. Latanoprost 


\section{INTRODUÇÃO}

A latanoprosta é um análogo das prostaglandinas conhecido pelo seu uso no tratamento da hipertensão ocular e glaucoma (TOSTI et al., 2004). Durante o tratamento oftalmológico com o medicamento que tinha como função a promoção do aumento do fluxo úveo-escleral, foi observado que os pacientes que faziam o uso dessa solução apresentavam efeitos colaterais como hipertricose, com aumento do comprimento e da espessura dos cílios (CHIBA et al., 2004). A partir de então tal solução foi associada ao minoxidil e inclusa nos tratamentos para queda de cabelo (BLOCH et al., 2018).

As prostaglandinas são sintetizadas com a catalise das COXs adicionando oxigênio ao ácido araquidônico tendo como resultado as prostaglandinas $\mathrm{G}_{2}$ também conhecidas como PGG. Elas são chamadas de PGs primárias, por ainda terem pouca atividade, porém, elas são usadas como um substrato para a formação de outras PGs como $\mathrm{PGD}_{2}, \mathrm{PGE}_{2}, \mathrm{PGF}_{2} \alpha, \mathrm{PGI}_{2}$. A latanoprosta faz parte das prostaglandinas $\alpha$ onde a prostaglandina $\mathrm{PGD}_{2}$ tem ação análoga à ação da latanoprosta. Quantidades elevadas de prostaglandinas $\mathrm{D}_{2}$ inibem o crescimento capilar, enquanto as prostaglandinas do tipo $\mathrm{E}$ e do tipo $\mathrm{F}_{2}$-alfa, tem ação estimulatória no ciclo capilar (CHOI; DIEHL; LEVINS, 2014). Indivíduos que apresentam quadros de alopecia têm as duas prostaglandinas de efeito estimulador diminuídas ou até mesmo inexistentes. Esse processo ocorre devido a prostaglandina $\mathrm{F}_{2}$-alfa induzir a mitose e aumentar a transcrição de fatores nucleares levando a uma síntese de proteases que causam alterações na matriz extracelular e que podem ser precursores na transformação de pelos vêlus em pelos terminais (TORIS; GABELT; KAUFMAN,2008). Além disso, a latanoprosta é uma vasodilatadora que ativa a melanogênese do folículo capilar, inibindo a apoptose celular e prolongando o ciclo capilar (NESTE; TOBIN,2004).

Estudos de caso-controle em primatas, associando um grupo com latanoprosta e outro com medicações a base de fosfato, apresentaram um crescimento capilar acentuado nos grupos que receberam a latanoprosta (UNO et al., 2002). Ensaios clínicos em humanos já estão sendo realizados (FAGHIHI; ANDALIB; ASILIAN, 2009). Mas a maioria ainda investiga o efeito da latanoprosta no crescimento ciliar (CHIBA et al., 2004; FAGHIHI; ANDALIB; ASILIAN, 2009). Alguns efeitos adversos são relatados com o uso do medicamento como vermelhidão nos olhos, hiperpigmentação da íris e prurido no local de aplicação (MUKHOPADHYAY; PLUMB, 2008; MEHTA et al., 2003). Contudo esses efeitos são esporádicos e não ocorrem em todos os indivíduos (BELLAND et al., 2011). 
Existem vários tipos de alopécia, cada uma com a sua característica, causa, diagnóstico e forma de tratamento. As mais comuns são a alopécia areata, eflúvio telógeno e a alopécia androgenética.

Na alopécia areata (AA) é uma patologia dos folículos pilosos e das unhas. É considerada idiopática, porém sabe-se que está associada a situações de estresse. Tem como característica principal a queda repentina dos pelos, deixando falhas em formato de círculos na região afetada em alguns indivíduos pode acontecer o desaparecimento por completo dos fios, mas em sua maioria o pelo volta a crescer e as falhas são preenchidas (CARDOZO et al, 2016). O diagnóstico da AA comumente é clínico, mas em casos mais graves são necessárias biopsias e exames auxiliares como o tricograma do couro cabeludo. O tratamento mais utilizado para esse tipo de alopécia é a base de corticosteroides (MAIA; FERNANDES, 2003).

O eflúvio telógeno tem como característica principal a queda excessiva dos cabelos que estão na fase telógena decorrente da anormalidade no ciclo capilar. O eflúvio telógeno apresenta fase aguda e crônica. Na fase aguda caem em média de 100 a 200 fios de cabelo por dia por um período de até seis meses. Nestes casos a alopécia não é tão perceptível, porque $50 \%$ dos cabelos estão sendo perdidos antes mesmo que ocorra uma redução aparente da densidade capilar (SILVA; SANCHEZ; PEREIRA, 2011) Na fase crônica do eflúvio telógeno também conhecido como alopécia difusa, as mulheres são as mais acometidas, principalmente na faixa etária de 30 a 50 anos, em que a queda capilar permanece por mais de seis meses (SILVA; SANCHEZ; PEREIRA, 2011) As causas do eflúvio telógeno podem ser por deficiências nutricionais, uso de medicações, distúrbios hormonais, lúpus, gestação e problemas endócrinos como o hipotireoidismo ou hipertireoidismo. O tratamento para o eflúvio telógeno depende da causa, mas também é indicado o uso de corticosteroides e soluções que promovam a vaso dilação do tecido. Neste tipo de alopécia não ocorre uma calvície completa, mas uma rarefação do cabelo (PEREIRA, 2006).

A alopécia androgenética (AGA) é uma calvície geneticamente determinada com característica de transmissão autossômica dominante. A AGA é a forma mais comum de queda capilar em ambos os sexos (MULINARI-BRENNER; SOARES, 2009). O diagnóstico da AGA geralmente é clínico, contudo alguns testes podem auxiliar como exames físicos, teste de tração e o uso de lupas especificas. Em casos raros pode ser necessário a realização de uma biopsia e ou um tricograma de couro cabeludo (MULINARI-BRENNER; SEIDEL; HEPP, 2011). 
O ciclo capilar é composto de três fases de desenvolvimento as fases anágena, catágena e telógena tem duração em média de cinco anos. Cada folículo piloso tem o seu próprio ciclo de desenvolvimento, desta forma em um mesmo período de tempo podemos ter folículos em variáveis fases (PEREIRA, et al., 2018).

A fase anágena ou hiperploriferativa é a fase mais longa de todo o ciclo, ela pode durar alguns anos e mais da metade dos folículos se encontram nela. Os folículos ficam envolvidos por capilares sanguíneos que levam nutrientes e substancias exógenas que serão incorporadas pelo restante do fio. É na fase anágena que ocorre a formação da estrutura do fio e da cutícula (AUDI et al., 2017).

Na fase catágena o cabelo já não recebe mais irrigação sanguínea e a divisão celular é interrompida definitivamente. É na fase catágena que o fio para de crescer e morre, também é nesta fase que a atividade dos melanócitos é cessada e o folículo se recolhe deixando na superfície da camada, um saco repleto de células potenciais de papila dérmica, para que o bulbo fique ativo. Somente $1 \%$ dos fios se encontram nessa fase que dura somente algumas semanas (MELLO, 2010).

A fase telógena também é conhecida como fase de repouso, nela ocorre a expulsão do folículo pelo desprendimento do fio do couro cabeludo, ou seja, é a fase final do ciclo de vida do cabelo. A queda desse fio ocorre na lavagem dos cabelos, esfregaço do couro cabeludo e ao se pentear os fios. Esse processo determina o fim de um ciclo e logo em seguida, início do outro, com o desenvolvimento e crescimento de um novo folículo de fase anágena (AUDI et al., 2017). Temos nesta fase, cerca de 10 a 14\% dos folículos e duração média de três a quatro meses (MAIO, 2004).

Os tratamentos realizados com a latanoprosta costumam durar de três meses a dois anos (MANSBERGER; CIOFFI, 2000). E sua composição geralmente varia em 0,0005\% a $0,5 \%$, com administração na maioria das vezes por via tópica (PEREZ; REY; MARTÍNEZ,2010; STECCHI et al., 2002). No entanto, o mecanismo de ação dos ativos envolvidos no crescimento capilar como os da latanoprosta, ainda não estão totalmente esclarecidos e o que se sabe é que a latanoprosta parece estimular a fase anágena e aumentar a conversão de pelos vêlus em pelos terminais (BLOCH et al., 2018).

Apesar do papel do minoxidil já ser bem estabelecido no crescimento capilar, novos medicamentos como a latanoprosta precisam ser investigados para possível aplicação em tratamentos capilares como em casos diagnosticados com alopecia areata e androgenética. Com isso, este trabalho tem por objetivo revisar através da literatura o papel da latanoprosta no crescimento de pelos para possível uso na terapia capilar. 


\section{METODOLOGIA}

Trata-se de uma revisão descritiva da literatura que buscou evidenciar e discutir o papel da latanoprosta no crescimento de pelos. A busca e coleta dos dados foi realizada por meio de protocolo de busca, elaborado pelos autores. Foram pré-estabelecidos e delimitados: tema de interesse, critérios de inclusão, estratégias de busca e seleção, formulário para obtenção dos dados colhidos, análise e apresentação dos resultados e interpretação dos resultados dos estudos.

\subsection{Critérios de inclusão e exclusão}

Foram incluídos artigos completos que avaliaram o uso da latanoprosta como estimulador do crescimento de pelos. Foram excluídos todos os estudos que investigaram apenas o papel da latanoprosta no tratamento de glaucoma ou patologias não relacionadas à pelos ou tecido capilar.

\subsection{Estratégia de busca e seleção}

Foram analisadas publicações indexadas na base MEDLINE, consultadas por meio do PUBMED; Biblioteca Virtual de Saúde (BVS), Periódicos Capes e Scientific Eletronic Library Online (SCIELO), nos meses de junho de 2018 e julho de 2018, não houve delimitação por período ou língua.

O processo de localização e seleção dos artigos foi conduzido por dois pesquisadores de forma independente e um terceiro pesquisador finalizou o processo de tomada de decisão. Foram utilizados os seguintes termos de pesquisa: latanoprost AND hair, para BVS, SCIELO e Periódicos Capes. Para o PUBMED os seguintes termos MESH: (latanoprost OR xalatan) OR PhXA34) OR PhXA41) AND hair). Para os Periódicos Capes os termos foram filtrados por assunto e modalidade de artigo.

Procedeu-se a busca manual na lista de referências de artigos de revisão e artigos localizados com essa estratégia de busca, para garantir a inclusão de todos os artigos relevantes ao tema. As publicações em duplicatas foram removidas manualmente.

\subsection{Extração dos dados}

Para cada estudo incluído, foram extraídos autor; periódico; ano de publicação; região do estudo; tamanho da amostra; local de uso; concentração e eficácia do medicamento; 
forma de aplicação; características do crescimento; duração e indicação do tratamento. Todos os resultados encontrados foram descritos em tabelas.

\section{RESULTADOS E DISCUSSÃO}

De 111 artigos relacionados com a latanoprosta e o crescimento de pelos, apenas nove foram incluídos nesta revisão. A figura 1 apresenta o fluxograma de busca e seleção dos artigos.

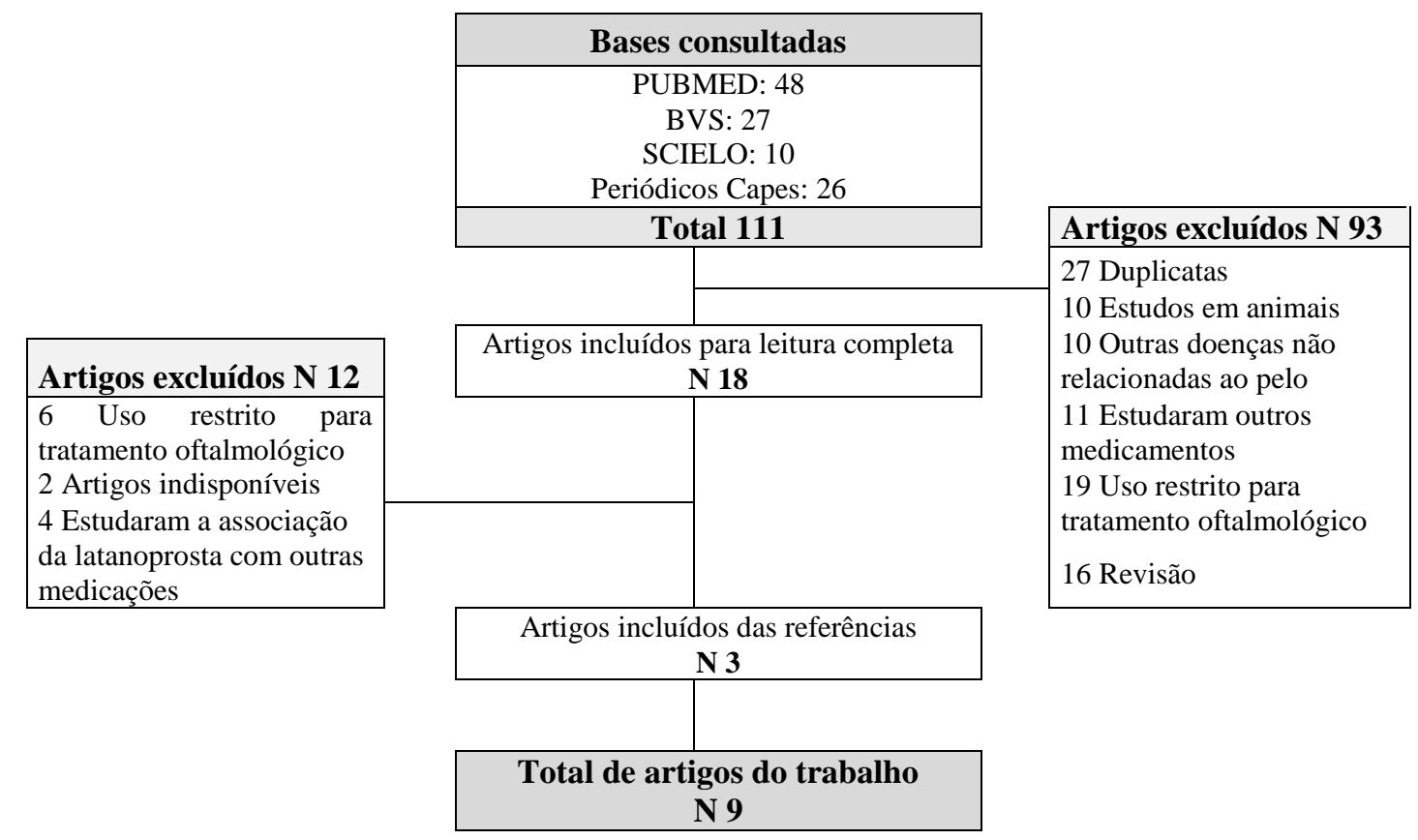

Figura 1. Fluxograma da busca e seleção dos artigos incluídos na revisão.

A latanoprosta é um medicamento com efeito já bem conhecido no tratamento do glaucoma (RUSSO et al., 2008). A hipertricose ciliar foi observada como efeito adverso da droga (RUSSO et al., 2008), e despertou o interesse de profissionais como dermatologistas e esteticista, para uso em tratamentos capilares, principalmente para as alopecias (SILVA; SANCHEZ; PEREIRA, 2011). Não há ainda na literatura, um consenso sobre a influência da latanoprosta no crescimento de pelos.

Apesar de recente o uso da latanoprosta no tratamento de queda de pelos, nosso estudo levantou uma serie de publicações que observaram os seus efeitos no crescimento de pelos. A maioria dos estudos eram do tipo caso-controle com avaliação do uso da latanoprosta principalmente no crescimento de pelos do couro cabeludo e cílios com alopecia. O número de participantes dos estudos variou de um a 54 indivíduos e a medicação foi utilizada principalmente por via tópica na concentração de $0,005 \%$ (tabela 1). Infelizmente, nem todos 
os trabalhos incluídos avaliaram a eficácia da latanoprosta ou descreveram adequadamente a maneira como foi avaliado o crescimento dos pelos (tabela 2). 


\begin{tabular}{|c|c|c|c|c|}
\hline Autor & Eficácia* & $\begin{array}{c}\text { Tempo } \\
\text { tratamento }\end{array}$ & Efeitos adversos & Efeitos no pelo \\
\hline
\end{tabular}

2 Tabela 1. Características dos estudos incluídos que avaliaram o uso da latanoprosta no crescimento de pelos.

\begin{tabular}{c|c|c|c|c|c|c}
\hline Autor & Local & Tipo de Estudo & N & Origem do pelo & Tipo de medicamento & Tratamento* \\
\hline Belland et al., 2011 & Itália & Estudo de caso & 1 & Couro cabeludo & Latanoprosta tópico 0,005\% & RC \\
\hline Block et al., 2018 & Brasil & Caso-controle & 50 & Couro cabeludo & $\begin{array}{c}\text { Latanoprosta tópica } 0,005 \% \\
\text { Latanoprosta tópica } 0,010 \%\end{array}$ \\
\hline Faghihi et al., 2009 & Iran & Caso-controle & 26 & Cílios e sobrancelha & Latanoprosta tópica $0,005 \%$ & AA \\
\hline Mehta et al.,2004 & Reino unido & Estudo de caso & 1 & Cílios & Latanoprosta tópica (ND)** & AA \\
\hline Perez et al., 2009 & Espanha & Caso-controle & 54 & Ć́lios & Latanoprosta tópica $0.005 \%$ & AA \\
\hline Peytavi et al., 2011 & Alemanha & Caso-controle & 16 & Couro cabeludo & Latanoprosta tópica $0,1 \%$ & AD \\
\hline Ross et al., 2005 & Canadá & Ensaio clínico prospectivo & 11 & Sobrancelha & Latanoprosta tópica $(3 \mu \mathrm{g} / \mathrm{d})$ & AA \\
\hline Stecchi et al., 2002 & Roma & Caso-controle & 44 & Cílios & Latanoprosta tópica $0,005 \%$ & AA \\
\hline Vaccaro et al.,2015 & Itália & Estudo de caso & 1 & Couro cabeludo & Latanoprosta tópica $0,1 \%$ & AD \\
\hline
\end{tabular}

*Tratamento AD: Alopécia androgenética; AA: Alopéciaareata; RC: Repigmentação capilar induzida; ** ND: Concentração não disponível. 


\begin{tabular}{|c|c|c|c|c|}
\hline Belland et al., 2011 & NA & 3 anos & Não houve efeito adverso & $\begin{array}{l}\text { Escurecimento do cabelo branco; Sobrancelhas e a pele ao redor dos olhos } \\
\text { inalterados }\end{array}$ \\
\hline Block et al.,2018 & $\begin{array}{c}\text { Não } \\
\text { avaliada }\end{array}$ & 6 meses & Não houve efeito adverso & $\begin{array}{l}\text { Grupo caso } 1 \text { (uso de latanoprosta a } 0,05 \% \text { ): Aumentou do número total de } \\
\text { fios; Grupo caso } 2 \text { (latanoprosta a } 0,010 \% \text { ) e Grupo placebo: sem } \\
\text { diferença significativa de crescimento. }\end{array}$ \\
\hline Faghihi et al., 2009 & NA & 4 meses & Cefaleia temporária & $\begin{array}{l}\text { Grupo caso: Crescimento parcial dos pelos em } 3,85 \% \text {. Grupo controle: } \\
\text { Não houve crescimento dos pelos. }\end{array}$ \\
\hline Mehta et al.,2004 & NA & 6 meses & Não houve efeito adverso & Alto crescimento ciliar \\
\hline Perez et al., 2009 & $45 \%$ & 2 anos & Não houve efeito adverso & $\begin{array}{l}\text { Grupo controle (n10): Não houve resposta ao tratamento e crescimento de } \\
\text { pelos. Grupo caso (n44): Recrescimento completo (17,5\%); Crescimento } \\
\text { moderado }(27,5 \%) \text {; Crescimento leve (30\%); sem resposta após dois anos } \\
\text { de tratamento }(25 \%) \text {. }\end{array}$ \\
\hline Peytavi et al., 2011 & $50 \%$ & 6 meses & Não houve efeito adverso & $\begin{array}{l}\text { Grupo caso e controle no mesmo paciente. Foi observado um aumento da } \\
\text { densidade capilar no local tratado com latanoprosta em comparação com a } \\
\text { linha de base ( } n=16, p .001) \text { e local tratado com placebo (p.0,0004). }\end{array}$ \\
\hline Ross et al., 2005 & NA & 1 mês & $\begin{array}{l}\text { Queda de pálpebra superior, } \\
\text { prurido e eritema temporários }\end{array}$ & $\begin{array}{l}\text { Os resultados no crescimento da sobrancelha após o tratamento não } \\
\text { apresentaram efeito significativo. }\end{array}$ \\
\hline Stecchi et al., 2002 & $7 \%$ & 6 meses & Não houve efeito adverso & $\begin{array}{l}\text { Grupo caso (latanoprosta a } 0,005 \%, \mathrm{n} 26) \text { : ligeira tendência de } \\
\text { crescimento }(0,2 \mathrm{~mm}) \text {. Grupo controle (timolol a } 0,5 \%, \mathrm{n} 18) \text { : não houve } \\
\text { crescimento. Não houve resultados significativos para ambos os grupos. }\end{array}$ \\
\hline Vaccaro et al.,2015 & NA & 72 horas & Alergia ao medicamento & Não houve crescimento \\
\hline
\end{tabular}

1 Tabela 2. Características dos estudos incluídos em relação ao tempo de uso da latanoprosta e efeitos no indivíduo. *NA: Não avaliada; 
Foram incluídos cinco estudos de caso controle, destes, dois avaliaram o uso da latanoprosta em couro cabeludo (BLOCK et al., 2018; PEYTAVI et al., 2011). E dois em cílios e sobrancelha (PÉREZ; REY; MARTÍNEZ, 2010; STECCHI et al., 2002). Um dos estudos com o maior número de indivíduos foi realizado no Brasil, com pacientes em tratamento de alopecia androgenética (BLOCK et al., 2018).

A maior parte dos estudos incluídos avaliaram o uso da latanoprosta tópica no tratamento de alopecia areata, principalmente em cílios e sobrancelhas. Os resultados em sua maioria, apresentaram-se satisfatórios, com maior relevância nos estudos com grupos controles (ROSS et al., 2005; MEHTA et al., 2003), em que nos grupos casos foi observado o aumento dos pelos, da pigmentação, espessura e do crescimento.

A alopecia areata é idiopática com características bem marcantes, onde encontramos queda do cabelo em formato de círculos ao longo do couro cabeludo. Já a alopecia androgenética é mais comum do que a alopecia areata, pois, é causada pelo aumento da testosterona e por fatores genéticos que apresentam a rarefação do cabelo na região frontal do couro cabeludo (KEVIN et al., 2017). Nesta revisão apenas um estudo avaliou o uso da latanoprosta em tratamento de repigmentação capilar induzida (BELLAND et al., 2011).

O mecanismo subjacente da mudança de cabelo e pele associada a latanoprosta no crescimento e pigmentação é pouco compreendido. Como a latanoprosta é um agonista seletivo do receptor $\mathrm{PGF}_{2} \alpha$ é provável que o fenômeno seja mediado por esse receptor (STJERNSCHANTZ, 2001). Alguns estudos já comprovaram que as prostaglandinas interferem no ciclo capilar e que seu desequilíbrio ou suas funções, levam a uma drástica redução da fase anágena do fio e consequentemente o aumento das fases seguintes, catágena e telógena. $\mathrm{O}$ aumento do número de fios que caem e consequentemente a redução do crescimento, leva a miniaturização folicular com pelos terminais menores que pelos vêlus, ou seja, esses fios se tornam cada vez menores e mais finos (SASAKI; HOZUMI; KONDO, 2005; STAMPER; LIEBERMAN; DRAKE, 2009).

A latanoprosta aparentemente tem relação com a duração mais prolongada do crescimento do pelo, isso porque sua ação parece ter efeito nas papilas dérmicas, que são uma região extremamente vascularizada e que fazem a irrigação do bulbo capilar (WOLF et al., 2003). As papilas dérmicas estão mais envolvidas com a fase anágena do pelo e através delas ocorre a nutrição do bulbo capilar. Provavelmente, a latanoprosta é absorvida pela epiderme através das papilas e chega ao bulbo, que de alguma forma sofre os efeitos do medicamento aumentando a fase anágena e tornando o fio mais forte e espesso (DRISKELL et al., 2011). 
Os tratamentos avaliados nos estudos variaram entre 1 mês à 3 anos. Geralmente o ciclo do cabelo dura cerca de três a sete anos, sendo que a fase anátena é a fase mais longa de todo o ciclo, portanto um período menor que três meses não é suficiente para avaliar com qualidade o crescimento do pelo com o uso da latanoprosta. Uma das características que foi observada durante os tratamentos é que mesmo depois da suspensão do uso da latanoprosta o pelo não diminuía a fase anágena (RAMOS; MIOT,2015; PEREZ; REY; MARTÍNEZ, 2010). Diferente de outras medicações com a mesma finalidade que, após suspensão do uso o fio volta a cair (ASHMAWY; MAADAWY; MAGHRABY,2018; MOROI, 2010).

Um dos estudos incluídos nesta revisão relatou tempo de tratamento de 72 horas, contudo a paciente não chegou a finalizar o tratamento porque houve efeitos adversos a medicação no couro cabeludo (VACCARO et al., 2015).

Com base nos estudos incluídos nesta revisão, especulamos que o uso prolongado da latanoprosta pode proporcionar um resultado visivelmente mais eficaz do que um período curto de uso.

A concentração de uso da latanoprosta nos estudos investigados variam de $0,005 \%$ a $0,1 \%$. Soluções oftalmológicas comercializadas também apresentam as mesmas proporções (ANVISA, 2013). Um dos estudos incluídos observou que a latanoprosta usada nas proporções de 0,01\% (VACCARO et al., 2015), não gerou crescimento no pelo, mas outro estudo com a mesma proporção levou ao crescimento significativo do fio (p. 0,0004) (PEYTAVI et al., 2011), ambos os estudos foram no couro cabeludo. Sugerimos que talvez a proporção da droga aplicada também possa influenciar no desenvolvimento do fio e que uma padronização dessa proporção é necessária para eventuais estudos.

A maior parte das publicações não relatou efeitos adversos ao uso de latanoprosta, mas entre as implicações observadas houve quadros alérgicos (VACCARO et al., 2015) cefaleia temporária (FAGHIHI; ANDALIB; ASILIAN, 2009) e queda de pálpebra superior com prurido e eritema temporários (ROSS et al., 2005).

Este estudo não incluiu artigos que avaliaram o crescimento de pelos em indivíduos com glaucoma, por que entendemos que, a hipertricose observada foi um efeito não esperado da droga durante o tratamento. Contudo, é importante ressaltar que alguns estudos associados a latanoprosta intraocular relataram a hipertricose e o aumento da pigmentação dos cílios, principalmente quando o uso era maior que três meses (MANSBERGER; CIOFFI, 2000; SURGIMOTO E UJI, 2002; CHIBA et al., 2004; MONSELISE; SHAPIRO; LUI,2011; OZYURT; CETINKAYA, 2014). 
Para elucidar ainda mais os efeitos colaterais cutâneos do uso tópico da latanoprosta em tratamentos oftalmológicos, um estudo japonês examinou 317 pacientes com glaucoma que fizeram o uso da latanoprosta uma vez ao dia durante aproximadamente quatro meses e verificaram que $77 \%$ desses pacientes desenvolveram hipertricose das pálpebras superiores e inferiores especialmente da pálpebra inferior lateral (DEMITSU et al., 2001).

O mecanismo de crescimento dos pelos na maioria dos estudos não é claro, mas é conhecida a característica dos folículos pilosos na expressão de receptores de prostaglandinas, que são receptores alvo do latanoprosta. Além disso, a prostaglandinas F2 alfa, são mitógenas e assim, estimulam o crescimento e a proliferação celular (HAKEDA et al., 1997). Por isso, ensaios clínicos direcionados para essas vias, elucidariam melhor a farmacocinética e farmacodinâmica do medicamento.

Não é possível ainda, dizer se a latanoprosta realmente pode ou não potencializar o crescimento de pelos, principalmente porque o número de artigos e amostragem é limitado. Contudo, também não podemos afirmar que o uso da droga em si pode levar a efeitos colaterais como os relatados. Acreditamos que, um ensaio clínico randomizado de caso controle, com um número maior de participantes, seria o ideal para comprovar a eficácia da latanoprosta.

Esta revisão incluiu somente estudos que fizeram uso da latanoprosta, todos os outros estudos que avaliaram a latanoprosta associada a outras medicações como o Minoxidil, Finasteride, Timolol e Bimatoprosta foram excluídas (MONSELISE; SHAPIRO; LUI,2011; CHOI; DIEHL; LEVINS, 2014). Entendemos que, apesar de recente o uso desse medicamento, seria interessante apenas descrever as alterações observadas com o uso da latanoprosta.

Um dos estudos avaliados incluiu 123 casos de alopecia androgênica no couro cabeludo que fez uso da latanoprosta, minoxidil e placebo, contudo foram apresentados na tabela apenas os grupos que receberam somente a latanoprosta e o grupo placebo (BLOCK et al., 2018). Neste estudo, a latanoprosta foi menos eficaz que o minoxidil e as proporções maiores que $0,005 \%$ da medicação não tiveram resultado significativo.

Entre as limitações deste estudo, destacamos a carência de trabalhos que investiguem o papel da latanoprosta como possível alvo terapêutico para o crescimento de pelos, principalmente em populações maiores. Além disso, a maioria dos estudos incluídos não apresentavam metodologia clara para avaliação do crescimento capilar, da eficácia da latanoprosta ou da forma e tempo de uso. Apesar desse viés, este estudo fornece importantes informações sobre os efeitos positivos do latanoprosta no crescimento de pelos e pode apoiar 
a abordagem de novos trabalhos que procuram usar análogos de prostaglandina como alvo para recrutamento de folículos capilares de pelos vêlus e terapias capilares para melhora das alopecias.

\section{CONCLUSÃO}

Apesar das poucas publicações encontradas é possível observar que, a latanoprosta aparentemente tem um papel importante no aumento do crescimento de pelos, principalmente estimulando a fase anágena do ciclo capilar. Contudo, ainda não temos dados suficientes para afirmar que ela será eficaz na terapia capilar. Mais pesquisas são necessárias para determinar a eficácia e segurança, bem como a dose ideal e duração do tratamento de latanoprosta ou outro candidato dos análogos das prostaglandinas. Vale ressaltar que, os artigos aqui revisados em sua maioria relacionavam o uso da latanoprosta no tratamento de alopecia areata e que seria interessante mais estudos com a alopecia androgenética. 


\section{REFERÊNCIA}

ANVISA. Agência Nacional de Vigilância Sanitária. Aloxidil. 2013. Disponível em: < http://www.anvisa.gov.br/datavisa/fila_bula/frmVisualizarBula.asp?pNuTransacao $=38307620$ 13\&pIdAnexo=1611453>. Acessoem: 12 nov. 2018.

ASHMAWY,A.A.;MAADAWY,I.H.;MAGHRABY,G.M. Efficacy of topical latanoprost versus minoxidil and betamethasone valerate on the treatment of alopecia areata.J

DermatologTreat., v.29, n.1, p.55-64,2018. Disponível em:

<https://www.ncbi.nlm.nih.gov/pubmed/28521549> Acesso em:13 nov.2018.

AUDI, C.; et al. Desenvolvimento e mecanismo de ação da canície e queda capilar. Iniciação Revista de Iniciação Científica, Tecnológica e Artística Edição Temática em Saúde e Bem estar. São Paulo: Centro Universitário Senac. V. 6, N 5, abr., 2017.

BELLAND, S et al. Repigmentation of hair after latanoprost therapy. Journal of the European Academy of Dermatology and Venereology.,München, v.25, n.12,p.1485-7, 2011. Disponível em: <https://www.ncbi.nlm.nih.gov/pubmed/21198953>Acesso em: 13 set. 2018.

BLOCH, L. D et al. Latanoprosta e minoxidil: Estudo duplocegocomparat.ivo, placebocontrolado no tratamento da queda de cabelos. Surg. Cosmet. Dermatol., Rio de Janeiro, v.10, n.1, p.41-5, 2018. Disponível em:

<http://www.surgicalcosmetic.org.br/detalhe-artigo/618/> Acesso em: 13 set. 2018.

CARDOZO, A. F.; et al. Alopecia areata e os tratamentos alternativos. São Paulo, (Trabalho de Pesquisa do Curso Superior em Tecnologia de Estética e Cosmetologia, apresentado ao Centro Universitário das Faculdades Metropolitanas Unidas - FMU), 2016.

CHIBA, T et al. A Prospective Study of Iridial Pigmentation and Eyelash Changes Due to Ophthalmic Treatment with Latanoprost. Jpn J Ophthalmol., Yamanashi, v.48, p.141-147, 2004.Disponível em: <https://www.ncbi.nlm.nih.gov/pubmed/15060793 > Acesso em: 13 set. 2018.

CHOI, Y.M.; DIEHI, J.; LEVINS, P.C. Promising alternative clinical uses of prostaglandin F2 $\alpha$ analogs: beyond the eyelashes. J AmAcadDermatol., v.72, n.4, p.712-6, 2015.

Disponível em: <https://www.ncbi.nlm.nih.gov/pubmed/25601618> Acesso em: 03 set. 2018.

DEMITSU, D et al. Hypertrichosis Induced By Latanoprost. J Am AcadDermatol., v.44, n.4, p.721-3, 2001. Disponível em: <https://www.ncbi.nlm.nih.gov/ pubmed/11260563> Acesso em: 10 out. 2018. 
DRISKELL, R.R et al. Hair follicle dermalpapilla cells at a glance. JournalofCell Science., v.12a, p.1179-1182, 2011. Disponível em: <http://jcs.biologists.org/content/ 124/8/1179> Acesso em: 02 nov. 2018.

FAGHIHI, G.; ANDALIB, F.; ASILIAN, A. The efficacy of latanoprost in the treatment of alopecia areata of eyelashes and eyebrows. Eur J Dermatol.,Isfahan, v.19, n.6, p.586-587, 2009.Disponível em:<https://www.ncbi.nlm.nih.gov/pubmed/19620039 > Acesso em: 13 set. 2018.

HAKEDA, Y. et al.Prostaglandin F2a Stimulates Tyrosine Phosphorylation and Mitogen-Activated Protein Kinase in OsteoblasticMC3T3-E1 Cells via Protein Kinase C Activation.Endocrinology.,v.138, n.5, p.1821-1828.Disponível em: <https://academic.oup.com/endo/article/138/5/1821/2987455\#59152467> Acesso em: 19 nov.2018.

KEVIN, R et al. Hormonal therapy in female pattern hair loss. Int J WomensDermatol., v.3, n.1, p.53-57, 2017. Disponível em: <https://www.ncbi.nlm.nih.gov/ pmc/articles/PMC5419033/> Acesso em: 22 out. 2018.

MAIA, C. P. A.; FERNANDES, N. C. Tratamento da alopecia areata com corticóide tópico: estudo prospectivo randomizado duplo cego em crianças. An. Bras. Dermatol, Rio de Janeiro, V. 78, N 1, P. 63-71, jan./fev., 2003.

MAIO, M. Tratado de Medicina Estética. São Paulo: Ed Roca, 1 ed., V. 1, 2004.

MANSBERGER, S.; CIOFFI, S. Eyelash formation secondary latanoprosta treatment in a patient with alopecia. Archophthalmol., Portland, v.118, p.718, 2000.Disponível em: <https://www.ncbi.nlm.nih.gov/pubmed/10815168> Acesso em: 13 set. 2018.

MEHTA, J.S et al. Cutaneous latanoprost in the treatment of alopecia areata. Eye., v.17, n.3, p.444-446, 2003.Disponível em: <https://www.ncbi.nlm.nih.gov/pubmed/12724722> Acesso em: 13 set. 2018.

MELLO, M. S. A evolução dos tratamentos capilares para ondulações e alisamentos permanentes. Porto Alegre, 2010. 38f. (Trabalho de conclusão de curso da disciplina de Estágio Curricular em Farmácia Faculdade de farmácia departamento de produção e controle de medicamentos disciplina de Estagio curricular em Farmacia, Universidade Federal do Rio grande do sul), Rio Grande do Sul, 2010. 
MONSELINE,A.; SHAPIRO,J.; LUI,H. Inner canthus hypertrichosis: a side effect of prostaglandin analogue treatment for glaucoma. J Cutan Med Surg., v.15, n.5, p.2989,2011.Disponível em:< https://www.ncbi.nlm.nih.gov/pubmed/21962192> Acesso em: 04 nov.2018.

MOROI, S.E.Eyelash preservation during chemotherapy and topical prostaglandin therapy.ArchIntern Med., v.170, n.14, p.1269-70, 2010.Disponivel em: <https://www.ncbi.nlm.nih.gov/pubmed/20660849> Acesso em: 13 nov.2018.

MULINARI-BRENNER, F.; SOARES, I. F. Alopécia androgenética masculina: uma atualização. Rev. Ciênc. Méd., Campinas, V. 18, N 3, P. 153-161, mai./jun., 2009.

MULINARI-BRENNER, F.; SEIDEL, G.; HEPP, T. Entendendo a alopécia androgenética. Surg Cosmet Dermatol. Curitiba, V 3, N 4, P 329-37, 2011.

MUKHOPADHYAY, R.; PLUMB, A. A rare complication from prostaglandin analogue therapy. ClinExpOptom., United Kingdom, v.92, n.2, p.137-138, 2009.Disponível em: <https://www.ncbi.nlm.nih.gov/pubmed/18651842 > Acesso em: 13 set. 2018.

NESTE, D.V.; TOBIN, D.J. Hair cycle and hair pigmentation: dynamic interactions and changes associated with aging. Micron.,Tournai, v.35, n.3, p.193-200, 2004. Disponível em: <https://www.ncbi.nlm.nih.gov/pubmed/15036274> Acesso em: 10 out. 2018.

OZYURT, S.; ÇETINKAYA, G.S. Hypertrichosis of the malar areas and poliosis of the eyelashes caused by latanoprost..ActasDermosifiliogr., v.106, n.1, p.74-5. Disponível em :<https://www.ncbi.nlm.nih.gov/pubmed/25065768> Acesso em: 04 set.2018.

PEREIRA, J. M. Eflúvio telógeno após dermatite de contato no couro cabeludo. An. Bras. Dermatol. V. 81, N 5 Supl 3, P. S288-9, 2006.

PEREIRA, C. M. et al. Princípios ativos cosméticos utilizados no tratamento da alopécia. Disponível em:

$<$ http://siaibib01.univali.br/pdf/Carlos\%20Manoel\%20Pereira\%20e\%20Hamilton\%20Azeved o\%20Aguiar.pdf>. Acesso em: 25 abr., 2018.

PEREZ, C.; REY, R.; MARTINEZ, C. Latanoprost in the treatment of eyelash alopecia in alopecia areatauniversalis. J Eur Acad Dermatol Venereol.,Seville, v. 24, p. 481-485, 2010. Disponível em: <https://www.ncbi.nlm.nih.gov/pubmed/20028444> Acesso: 13 set. 2018. 
PEYTAVI, U et al. A randomized double-blind placebo-controlled pilot study to assess the efficacy of a 24 -week topical treatment by latanoprost $0.1 \%$ on hair growth and pigmentation in healthy volunteers with androgenetic alopecia. J AmAcadDermatol.Germany., v.66, n.5, p.794-800, 2011.Disponível em:

<https://www.ncbi.nlm.nih.gov/pubmed/21875758>Acesso: 13 set. 2018.

RAMOS, M.P.;MIOT,A.H. Female Pattern Hair Loss: a clinical and pathophysiological review.AnBrasDermatol., v.90, n.4,p.529-543,2015.Disponivel em:

<https://www.ncbi.nlm.nih.gov/pmc/articles/pmc4560543/> Acesso: 13 nov.2018.

ROSS, E.K et al. Lack of efficacy of topical latanoprost in the treatment of eyebrow alopecia areata. J AmAcadDermatol.,Colombia, v.53, n.6, p.1095-6, 2005. Disponível em: <https://www.ncbi.nlm.nih.gov/pubmed/16310083> Acesso em: 22 set. 2018.

RUSSO, A et al. Latanoprost ophthalmic solution in the treatment of open angle glaucoma or raised intraocular pressure: a review. ClinOphthalmol., v.2, n.4, p.897-905, 2008. Disponível em: <https://www.ncbi.nlm.nih.gov/pubmed/19668444> Acesso em: 16 out. 2018.

SASAKI, S.; HOZUMI, Y.; KONDO, S. Influence of prostaglandin F2alpha and its analogues on hair regrowth and follicular melanogenesis in a murine model.Exp Dermatol., v.14, n.5, p.323-8, 2005. Disponível em: <https://www.ncbi.nlm.nih.

gov/pubmed/15854125> Acesso em: 01 nov. 2018.

SILVA, A.P.; SANCHEZ, A.P.; PEREIRA, J.M. The importance of trichological examination in the diagnosis of alopecia areata. AnBrasDermatol., v.86, n.5, p.1039-41, 2011. Disponível em: <https://www.ncbi.nlm.nih.gov/pubmed/22147056> Acesso em: 20 out. 2018.

\section{STAMPER,R.L.;LIEBERMAN,M.F.;DRAKE,M.V Becker-Shaffer's Diagnosis and}

Therapy of the Glaucomas.8.ed.EUA: Editora Copyright,2009. Disponível em: $<$ https://www.elsevier.com/books/becker-shaffers-diagnosis-and-therapy-of-theglaucomas/9780323023948> Acesso em: 19 nov.2018.

STECCHI, G et al. Eyelash hypertrichosis induced by topical latanoprost: 6-month follow-up study. Acta OphthalmologicaScandSuppl., v.236,p.56-7, 2002. Disponível em: <https://www.ncbi.nlm.nih.gov/pubmed/12390146> Acesso em: 22 set. 2018.

STJERNSCHANTZ, J.W. From PGF (2alpha)-isopropyl ester to latanoprost: a review of the development of xalatan: the Proctor Lecture.Invest Ophthalmol Vis Sci., v. 42, n.6, p.113445, 2001. Disponível em: <https://www.ncbi.nlm.nih.gov/pubmed/ 11328719> Acesso em: 01 nov. 2018. 
SUGIMOTO, M.; UJI, Y. Quantitative analysis of eyelash lengthening following topical latanoprost therapy. Can J Ophthalmol., v.37, n.6, p.342-5, 2002. Disponível em: < https://www.ncbi.nlm.nih.gov/pubmed/12422916> Acesso em: 10 out. 2018

TORIS, C.B.; GABELT.; KAUFMAN, P.L. Update on the mechanism of action of topical prostaglandins for intraocular pressure reduction. SurvOphthalmol., v.53, n.1, p.107-20, 2008. Disponível em: <https://www.ncbi.nlm.nih.gov/pubmed/19038618> Acesso em: 03 set. 2018.

TOSTI, A et al. Evaluation of sexual function with an international index of erectile function in subjects taking finasteride for androgenetic alopecia. ArchDermatol., v.140, n.7, p.857-8, 2004. Disponível em: <https://www.ncbi.nlm.nih.gov/pubmed/ 15262698> Acesso em: 29 agos. 2018.

UNO, H et al. Effect of Latanoprost on Hair Growth in the Bald Scalp of the Stump-tailed Macacque: A Pilot Study. Acta DermVenereol., v.82, n.1, p.7-12, 2002.Disponível em: <https://www.ncbi.nlm.nih.gov/pubmed/12013211> Acesso em: 01 out. 2018.

VACCARO, $\mathrm{M}$ et al. Erosive pustular dermatosis of the scalp following topical latanoprost for androgenetic alopecia.DermatolTher., v.28, n.2, p.65-7,2015. Disponivel em:<https://www.ncbi.nlm.nih.gov/pubmed/25627907> Acesso em: 10 nov.2018.

WOLF, R et al. Prostaglandin analogs for hair growth: great expectations. Dermatol Online J.,v.9,n.3,p.7,2003. Disponível em:<https://www.ncbi.nlm.nih.gov/pubmed/ 12952754> Acesso em:18 nov. 2008. 